\title{
The microbiome adds to the complexity of parathyroid hormone action on bone
}

\author{
Sundeep Khosla \\ Division of Endocrinology and Kogod Center on Aging, Mayo Clinic College of Medicine, Rochester, Minnesota, USA
}

\begin{abstract}
Parathyroid hormone (PTH) has complex effects on bone, including stimulating bone formation and regulating the hematopoietic stem cell (HSC) niche. In the current issue of the $J \mathrm{Cl}$, Li et al. demonstrated that the microbiome, through the production of short-chain fatty acids and in particular, butyrate, is necessary for the ability of PTH to increase osteoblast numbers and stimulate bone formation. In addition to implications for the treatment of osteoporosis with PTH analogs, this pathway may be part of a broader mechanism through which the microbiome serves its key function of modulating the immune system.
\end{abstract}

\section{PTH actions on bone}

Parathyroid hormone (PTH) is the most critical factor regulating plasma calcium levels and calcium homeostasis, but decades of investigation dating back to the seminal studies of Fuller Albright in the 1940s (1) have identified the pleiotropic physiological actions of PTH beyond this key role. These include regulation of bone formation and resorption, renal calcium as well as phosphorus handling, and more recently, the hematopoietic stem cell (HSC) niche $(2,3)$. Underpinning the complex physiological actions of PTH is an even greater complexity of target cells and genes that are involved in mediating the effects of PTH in various tissues. And for those attempting to build a clear understanding of precisely how PTH works, life just got more difficult, as in this issue of the JCI the Pacifici laboratory identifies the microbiome as a major regulator of the anabolic effects of PTH on bone (4).

Under physiological conditions of pulsatile PTH secretion or when PTH is administered exogenously on an intermittent basis (e.g., single daily injections), PTH sOtimulates bone formation to a greater extent than bone resorption. By contrast, exposure of bone to continuously elevated circulating PTH concentrations, as in primary hyperparathyroidism, leads to an excess of bone resorption over bone formation and net bone loss (2). Even limiting the discussion to the anabolic skeletal actions of PTH, it is now clear that PTH acts not on one, but on numerous target cells, although cells of the osteoblast lineage (osteoprogenitors, osteoblasts, osteocytes, and bone lining cells) have generally been considered the primary cells mediating PTH effects on bone (2). Thus, deletion of the PTH receptor (PTH1R) in osteocytes $(5,6)$ or the $G$ protein $\alpha$-subunit (which mediates PTH1R signaling) in osteoprogenitor cells (7) blunts the anabolic actions of PTH on bone. However, cells beyond those from an osteoblast lineage are also involved in PTH-induced bone formation and loss. For example, work from the McCauley laboratory has shown that MAFIA mice, which lack macrophages, also have an attenuated increase in bone volume fraction in response to PTH (8). This leads to the conclusion that PTH effects on bone are perhaps best considered in a model that involves a network of codependent pathways in multiple cell

Related Article: p. 1767

Conflict of interest: The author has declared that no conflict of interest exists.

Copyright: () 2020, American Society for Clinical Investigation.

Reference information: / Clin Invest. 2020;130(4):1615-1617. https://doi.org/10.1172/JCI135712.

types, whereby elimination of key nodes that may include specific cell types and/ or genes in this network renders the others ineffective, resulting in an attenuation or loss of PTH effects on bone.

For a number of years, the Pacifici laboratory has pursued one of these nodes in the network of PTH actions on bone, with a focus on those that involve $\mathrm{T}$ cells. The researchers initially demonstrated that intermittent administration of PTH increased the production of Wnt1Ob (which induces the expansion and differentiation of osteoprogenitor cells, ref. 9) in bone marrow $\mathrm{CD} 8^{+} \mathrm{T}$ cells and induced these immune cells to activate Wnt signaling in osteoprogenitor cells (10). Furthermore, PTH failed to have an anabolic effect on bone in mice lacking $\mathrm{T}$ cell-produced Wnt10b. In subsequent studies, the researchers established that PTH acted directly on $\mathrm{T}$ cells by demonstrating that the anabolic effect of PTH on bone was blunted in mice with deletion of PTH1R specifically in T cells (11).

\section{Microbiome effects on PTH action in bone}

In this issue of the JCI (4), Li and colleagues define steps relating to the microbiome that are upstream of $\mathrm{T}$ cell $\mathrm{Wnt} 1 \mathrm{Ob}$ production. Through a series of elegant studies, they identify butyrate (a short-chain fatty acid, SCFA) produced by gut microbiota as the key mediator of the microbiome effects on PTH action in bone. To explore the contribution of the microbiome, the authors used germ-free mice or wild-type mice treated with broad-spectrum antibiotics to deplete the microbiome and consequently inhibit the production of butyrate. They then showed that further treating these butyrate-deficient mice intermittently with PTH failed to increase bone volume fraction or osteoblast numbers in trabecular bone. They went on to demonstrate that butyrate binds to its receptor, GPR43, on dendritic cells and also acts directly on $\mathrm{CD}^{+}{ }^{+} \mathrm{T}$ cells, independently of GRP43 sig- 


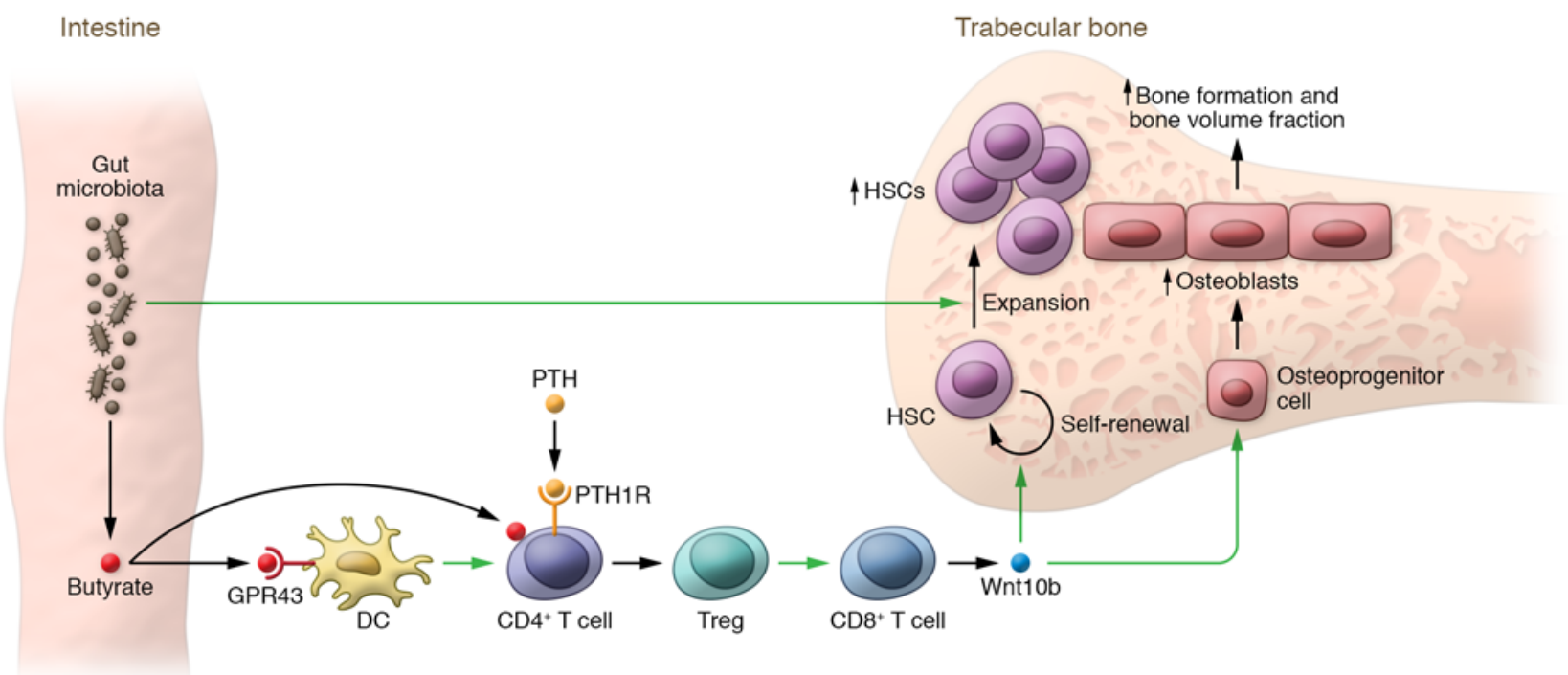

Figure 1. Model for interactions between the gut microbiome and PTH signaling, converging with HSC regulation. Butyrate produced by the gut microbiota binds to the GPR43 receptor on dendritic cells (DC) and also acts directly on CD4+ $4^{+}$cells independently of GPR43 signaling. PTH signals through the PTH1R on CD4+ $\mathrm{T}$ cells, and butyrate acts in concert with PTH to differentiate CD4+ $\mathrm{T}$ cells into Tregs which, in turn, stimulate CD8 ${ }^{+} \mathrm{T}$ cells to produce Wnt10b (4). Wnt10b acts on osteoprogenitor cells, leading to their expansion and differentiation (9), resulting in a net increase in osteoblasts on bone surfaces and increased bone formation as well as increased bone volume fraction. Osteoblasts also form part of the niche for the self-renewal and expansion of HSCs (3). In addition, the Wnt10b produced by T cells leads to the self-renewal of HSCs (14). Independent of this pathway, microbiota also lead to an expansion of HSCs (13), which require the osteoblast niche cells generated by the microbiome-PTH axis.

naling. While PTH signaled through the PTH1R on $\mathrm{CD}^{+}{ }^{+} \mathrm{T}$ cells, PTH also acted in concert with butyrate to induce $\mathrm{CD} 4^{+} \mathrm{T}$ cells to differentiate into regulatory $\mathrm{T}$ cells (Tregs) which, in turn, can stimulate $\mathrm{CD}^{+}$ $\mathrm{T}$ cells to produce Wnt10b. In addition, Wnt10b led to the expansion of osteoblasts on bone surfaces and increased bone formation (Figure 1). Based on these findings, $\mathrm{Li}$ et al. concluded that butyrate produced by gut microbiota is necessary for PTH to induce bone anabolism (4).

This is clearly an elaborate signaling mechanism whereby a SCFA produced by the gut microbiota acts on bone marrow dendritic cells, which then act on $\mathrm{CD} 4^{+} \mathrm{T}$ cells and, in concert with PTH, leads to an expansion of Tregs, which then signal to $\mathrm{CD}^{+} \mathrm{T}$ cells to stimulate osteoblastogenesis via Wnt10b. It is certainly reasonable to question the evolutionary advantage of this mechanism - it is unlikely that evolution designed this pathway in order to facilitate the efficacy of intermittent PTH administration as a treatment for osteoporosis in postmenopausal women. One potential explanation, which posits a link between the microbiome and the skeletal actions of PTH in regulating HSCs, is depicted in Figure 1. If we consider the major function of the microbiome - namely, modulation of the immune system (12) - and assess where it may intersect with what we know about the diverse actions of PTH, a common link that emerges is regulation of the HSC niche. Thus, a healthy microbiome plays a fundamental role in the induction, training, and function of the host immune system (12) and also leads to an expansion of HSCs, as treatment of mice with broad-spectrum antibiotics leads to a marked reduction in HSC numbers in bone (13). However, in order to expand HSCs, the microbiota also need to signal to bone to increase the number of HSC niche cells, specifically osteoblasts, that are critical for the self-renewal and expansion of HSCs (3). As depicted in Figure 1, SCFAs (butyrate) produced by microbiota may serve this purpose by acting in concert with one of the major regulators of osteoblast numbers in bone, PTH, to expand the very cells needed (i.e., osteoblasts) to sustain the HSC niche. This link may be even tighter than presently understood, as the Pacifici laboratory has previously shown that PTH itself increases the number of HSCs, at least in part by stimulating T cell Wnt1Ob, which acts not only on osteoprogenitors, but also on HSCs to stimulate their selfrenewal (14). Thus, through direct effects of microbial products on HSCs (13) and indirect effects via the butyrate-PTH axis, the microbiome simultaneously expands HSCs and their niche cells (osteoblasts) as part of its role in sustaining the immune system (Figure 1). In this formulation, the microbiome is likely oblivious to just how much bone its host has; rather, it is merely fulfilling its symbiotic contractual obligation to optimize the health of the host's immune system (12), and the stimulation of bone formation resulting from the microbiome/PTH-induced expansion of osteoblasts, which form at least part of the HSC niche (3), is a fortuitous consequence of the microbiome's main function related to the fortifying the immune system.

\section{Clinical implications}

In addition to providing informative mechanistic links between the microbiome, immune cells, and the skeletal actions of $\mathrm{PTH}$, the current findings also raise a number of important clinical questions. First, is there evidence for this microbiome-PTH link in humans? It would be important, for example, to test whether altering the microbiome in a positive (e.g., probiotics) or negative (e.g., broad-spectrum antibiotics) direction alters the skeletal response to the PTH analog, teriparatide, particularly in postmenopausal women in whom 
teriparatide is routinely used to treat osteoporosis (15). In addition, there is considerable variability in the skeletal anabolic response to teriparatide in terms of bone mineral density gains, including a subset of patients who fail to respond to teriparatide (16). Could this variable response or response failure be due, at least in part, to differences in the microbiome and/ or reduced circulating butyrate levels in these patients? Moreover, as suggested by the authors (4), could nutritional butyrate supplementation enhance the skeletal anabolic response to PTH in humans? Finally, based on the model in Figure 1, could butyrate, perhaps in concert with PTH, be useful in the recovery of HSCs following chemotherapy or in other settings of impaired hematopoiesis? As with any provocative preclinical study, the current work from the Pacifici laboratory (4) opens up new directions that will keep clinical investigators busy for a number of years and will, hopefully, lead to new treatment options for patients with osteoporosis and perhaps various hematological conditions involving a reduction in HSCs.

\section{Acknowledgments}

The author would like to thank his colleagues in the Mayo Kogod Center on
Aging for helpful review and comments. This work was supported by the National Institutes of Health, grants P01 AG062413, P01 AG004875, and R01 AG048792.

Address correspondence to: Sundeep Khosla, Mayo Clinic College of Medicine, 200 First Street SW, Rochester, Minnesota 55905, USA. Phone: 507.255.6663; Email: khosla.sundeep@mayo.edu.

1. Ingalls TH, Donaldson G, Albright F. The locus of action of the parathyroid hormone: experimental studies with parathyroid extract on normal and nephrectomized rats. JClin Invest. 1943;22(4):603-608.

2. Goltzman D. Physiology of parathyroid hormone. Endocrinol Metab Clin North Am. 2018;47(4):743-758.

3. Calvi LM, et al. Osteoblastic cells regulate the haematopoietic stem cell niche. Nature. 2003;425(6960):841-846.

4. Li J, et al. Parathyroid hormone-dependent bone formation requires butyrate production by intestinal microbiota. J Clin Invest. 2020;130(4):1767-1781.

5. Saini V, et al. Parathyroid hormone (PTH)/ PTH-related peptide type 1 receptor (PPR) signaling in osteocytes regulates anabolic and catabolic skeletal responses to PTH. J Biol Chem. 2013;288(28):20122-20134.

6. Delgado-Calle J, et al. Control of bone anabolism in response to mechanical loading and PTH by distinct mechanisms downstream of the PTH receptor. J Bone Miner Res. 2017;32(3):522-535.
7. Sinha P, et al. Loss of Gs $\alpha$ in the postnatal skeleton leads to low bone mass and a blunted response to anabolic parathyroid hormone therapy. J Biol Chem. 2016;291(4):1631-1642.

8. Cho SW, et al. Osteal macrophages support physiologic skeletal remodeling and anabolic actions of parathyroid hormone in bone. Proc Natl Acad Sci U S A. 2014;111(4):1545-1550.

9. Bennett $\mathrm{CN}$, et al. Wnt10b increases postnatal bone formation by enhancing osteoblast differentiation.J Bone Miner Res. 2007;22(12):1924-1932.

10. Terauchi M, et al. T lymphocytes amplify the anabolic activity of parathyroid hormone through Wnt10b signaling. Cell Metab. 2009;10(3):229-240.

11. Bedi B, et al. Silencing of parathyroid hormone (PTH) receptor 1 in T cells blunts the bone anabolic activity of PTH. Proc Natl Acad Sci US A. 2012;109(12):E725-E733.

12. Belkaid Y, Hand TW. Role of the microbiota in immunity and inflammation. Cell. 2014;157(1):121-141.

13. Josefsdottir KS, Baldridge MT, Kadmon CS, King KY. Antibiotics impair murine hematopoiesis by depleting the intestinal microbiota. Blood. 2017;129(6):729-739.

14. Li JY, et al. PTH expands short-term murine hemopoietic stem cells through T cells. Blood. 2012;120(22):4352-4362.

15. Khosla S, Hofbauer LC. Osteoporosis treatment: recent developments and ongoing challenges. Lancet Diabetes Endocrinol. 2017;5(11):898-907.

16. Gallagher JC, Rosen CJ, Chen P, Misurski DA, Marcus R. Response rate of bone mineral density to teriparatide in postmenopausal women with osteoporosis. Bone. 2006;39(6):1268-1275. 